\title{
Neurometabolic correlates of 6 and 16 weeks of treatment with risperidone in medication-naive first-episode psychosis patients
}

\author{
Badari Birur', Nina Vanessa Kraguljac (1), Lawrence VerHoef², Charity J. Morgan', Ripu Daman Jindal', \\ Meredith Amanda Reid (iD), Austin Luker ${ }^{1}$ and Adrienne Carol Lahti ${ }^{1}$
}

\begin{abstract}
Antipsychotic medications are the cornerstone of treatment in schizophrenia spectrum disorders. In first-episode psychosis, the recommended time for an antipsychotic medication trial is up to 16 weeks, but the biological correlates of shorter and longer antipsychotic treatment trials in these cohorts remain largely unknown. We enrolled 29 medication-naive first-episode patients (FEP) and 22 matched healthy controls (HC) in this magnetic resonance spectroscopy (MRS) study, examining the levels of combined glutamate and glutamine (commonly referred to as Glx) in the bilateral medial prefrontal cortex (MPFC) with a PRESS sequence (TR/TE $=2000 / 80 \mathrm{~ms}$ ) before initiation of antipsychotic treatment, after 6 and 16 weeks of treatment with risperidone. Data were quantified in $18 \mathrm{HC}$ and 20 FEP at baseline, for $19 \mathrm{HC}$ and $15 \mathrm{FEP}$ at week 6, and for $14 \mathrm{HC}$ and $16 \mathrm{FEP}$ at week 16. At baseline, none of the metabolites differed between groups. Metabolite levels did not change after 6 or 16 weeks of treatment in patients. Our data suggest that metabolite levels do not change after 6 or 16 weeks of treatment with risperidone in FEP. It is possible that our choice of sequence parameters and the limited sample size contributed to negative findings reported here. On the other hand, longer follow-up may be needed to detect treatment-related metabolic changes with MRS. In summary, our study adds to the efforts in better understanding glutamatergic neurometabolism in schizophrenia, especially as it relates to antipsychotic exposure.
\end{abstract}

\section{Introduction}

Antipsychotic medications are the foundation of acute and maintenance treatment in schizophrenia spectrum disorders. In multi-episode schizophrenia, effectiveness of the medication is typically established within $2-6$ weeks of treatment ${ }^{1-5}$. Recommendations for these timeframes as adequate duration of antipsychotic treatment trials are supported by meta-analyses demonstrating that the greatest fraction of antipsychotic response occurs within the first 2 weeks ${ }^{6,7}$, and that a substantial proportion of

Correspondence: Adrienne Carol Lahti (alahti@uabmc.edu)

'Department of Psychiatry and Behavioral Neurobiology, University of Alabama at Birmingham, Birmingham, AL, USA

${ }^{2}$ Department of Neurology, University of Alabama at Birmingham, Birmingham, $A L, U S A$

Full list of author information is available at the end of the article. symptom improvement after 1 year has already been achieved within the first 4 weeks ${ }^{7}$.

In first-episode psychosis, Gallego and colleagues reported that the cumulative response rate increased from $32 \%$ after 6 weeks of treatment to $65.2 \%$ after 16 weeks of treatment in first-episode patients ${ }^{8}$, arguing that treatment trials in first-episode psychosis should last several months. Unfortunately, the underlying mechanisms and biological correlates of short and longer treatment trails in this patient cohort remain largely unknown.

One method to investigate biological signatures of antipsychotic treatment is magnetic resonance spectroscopy (MRS), a non-invasive imaging technique used to study chemical composition, energy metabolism, and neurometabolites in vivo ${ }^{9}$. In the context of psychosis, examination of glutamatergic measures are of particular

\section{(c) The Author(s) 2020}

(c) Open Access This article is licensed under a Creative Commons Attribution 4.0 International License, which permits use, sharing, adaptation, distribution and reproduction cc) in any medium or format, as long as you give appropriate credit to the original author(s) and the source, provide a link to the Creative Commons license, and indicate if changes were made. The images or other third party material in this article are included in the article's Creative Commons license, unless indicated otherwise in a credit line to the material. If material is not included in the article's Creative Commons license and your intended use is not permitted by statutory regulation or exceeds the permitted use, you will need to obtain permission directly from the copyright holder. To view a copy of this license, visit http://creativecommons.org/licenses/by/4.0/. 
interest for several reasons. First, N-methyl-D-aspartate (NMDA) receptor hypofunction is thought to be a core feature of the pathophysiology ${ }^{10-14}$, possibly resulting in downstream dopaminergic dysfunction ${ }^{15-17}$. Second, several groups, including ours, have reported alterations in glutamatergic measures including glutamate, glutamine, and glutamine + glutamate (Glx) in schizophrenia $^{18-24}$. Third, a growing body of evidence suggests that antipsychotic treatment may decrease glutamatergic metabolite levels ${ }^{22,25-30}$, but also see a recent study by our group that did not find changes in glutamatergic metabolites in the anterior cingulate cortex and hippocampus after 6 weeks of antipsychotic treatment in patients with schizophrenia ${ }^{31}$.

The aim of our study was to examine longitudinally, neurometabolite levels in medication-naive first-episode psychosis (FEP) patients and changes after 6 and 16 weeks of treatment with risperidone, a commonly used secondgeneration antipsychotic medication. We chose the medial prefrontal cortex (MPFC) as our region of interest, because glutamatergic metabolites have been reported to be increased in this region in unmedicated, but not in medicated patients with schizophrenia ${ }^{22,26-30,32-35}$, suggesting that antipsychotic medications may affect glutamatergic measures in this area. Importantly, a recent study has reported that 4 weeks of treatment with risperidone resulted in a normalization of elevated MPFC Glx in medication-naive $\mathrm{FEP}^{36}$. Similarly, Egerton and colleagues reported a reduction in glutamate following treatment with amisulpride in $\mathrm{FEP}^{26}$.

Here, we scanned a group of antipsychotic-naive FEP, and then rescanned them after 6 and 16 weeks of treatment with risperidone to examine early and later medication effects on neurometabolite levels. We also scanned a group of matched healthy controls (HC) at the same intervals to assess the temporal stability of measurements. We hypothesized that ${ }^{1}$ Glx is increased, and that risperidone treatment ${ }^{3}$ decreases Glx. In an exploratory fashion, we also examined other commonly measured metabolites, including choline (Cho) and creatine $(\mathrm{Cr})$, and investigated relationships between metabolite levels and clinical variables.

\section{Methods}

Medication-naive FEP were recruited from the emergency room, inpatient units, and outpatient clinics at the University of Alabama at Birmingham (UAB). HC matched on age, sex, and parental occupation were recruited by advertisements. Approval for this study was obtained from the UAB Institutional Review Board and subjects provided written informed consent.

Diagnoses were established through consensus of two board certified psychiatrists (ACL and NVK) considering medical records and the Diagnostic Interview for Genetic
Studies (DIGS) ${ }^{37}$ as available. The Brief Psychiatric Rating Scale (BPRS) and Repeatable Battery for the Assessment of Neuropsychological Status (RBANS) were used to assess symptom severity and cognition ${ }^{38}$.

Subjects were excluded if they had major neurological or medical conditions, history of head trauma with loss of consciousness, substance use disorders (excluding nicotine) within 6 months of imaging, were pregnant or breastfeeding, or had MRI contraindications. Those with a positive drug screen were excluded. $\mathrm{HC}$ with a personal or family history in a first-degree relative of a psychiatric disorder were excluded.

Subjects who were antipsychotic-naive were enrolled in a 16-week trial of risperidone using a flexible dosing regimen. As part of a larger multimodal neuroimaging study, MRS of the MPFC was done prior to initiation of treatment, after 6 weeks of treatment, and again after 16 weeks of treatment. Risperidone was prescribed by ACL and NVK; it was started at 1-2 mg and titrated in 1-2 mg increments; dosing was based on therapeutic and side effects. Use of concomitant medications after risperidone was started was permitted as clinically indicated. Compliance was monitored with pill counts at each visit.

To obtain normative data, matched $\mathrm{HC}$ were scanned at the same three time points as the FEP patients. Overall, we enrolled $29 \mathrm{FEP}$ and $22 \mathrm{HC}$ in this study.

\section{Image acquisition}

Imaging was performed on a 3T head-only scanner (Magnetom Allegra, Siemens) using a circularly polarized transmit/receive head coil. A high-resolution structural scan was acquired (MPRAGE, TR/TE/TI $=2300 / 3.93$ / $1100 \mathrm{~ms}, 1 \mathrm{~mm}$ isotropic voxels).

A series of T1-weighted anatomical scans $(\mathrm{TR} / \mathrm{TE}=$ 250/3.48 ms, 5-mm slice thickness) were acquired to aid placement of the bilateral MPFC $(20 \times 20 \times 20 \mathrm{~mm})$. Voxel placement for the second and third acquisition was guided by an image of the voxel placement during the first scan. After manual shimming, CHESS pulses were used to suppress the water signal. Spectra were acquired using a PRESS sequence $(T R / T E=2000 / 80 \mathrm{~ms}$, to optimize the glutamate signal $^{39}$ and minimize macromolecule contribution, $1200 \mathrm{~Hz}$ spectral bandwidth, 1024 points, 256 averages). Eight averages of unsuppressed water with the same sequence parameters were also acquired immediately afterwards to use as a reference. Examples of voxel placement and spectra are depicted in Fig. 1. To limit effects of nicotine intoxication or withdrawal, participants were allowed, but not encouraged, to smoke up to one hour before acquisition of images.

\section{MRS data processing}

MRS data were quantified in the time domain with AMARES in jMRUI (version 5.2); prior knowledge 

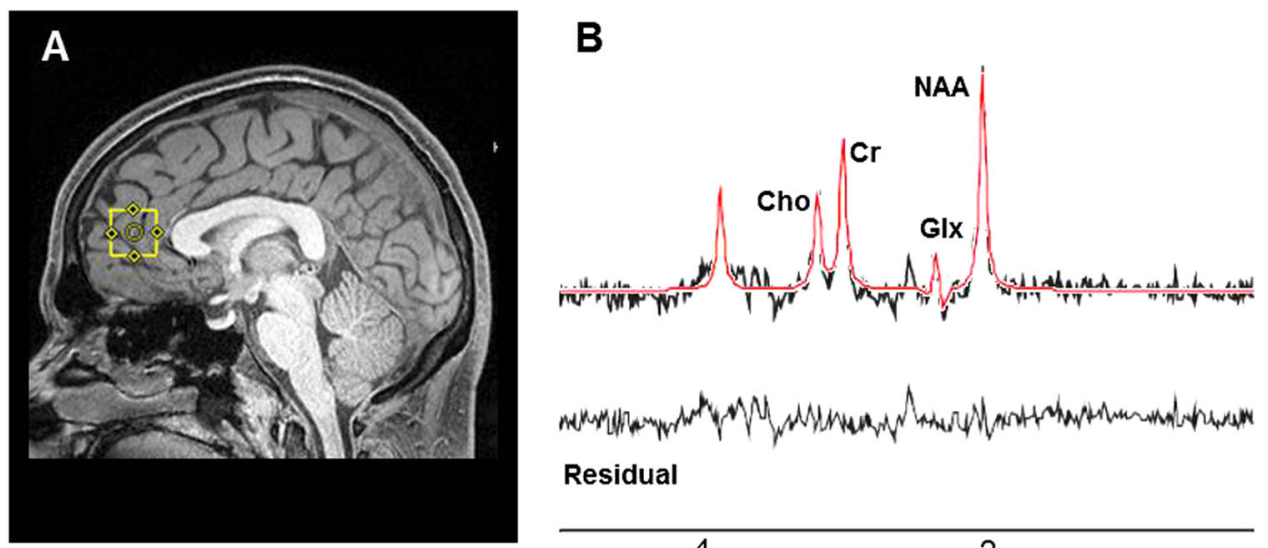

Residual

4 2

ppm

Fig. 1 Illustration of voxel placement and example spectrum. a Voxel placement in the medial prefrontal cortex $(2.0 \times 2.0 \times 2.0 \mathrm{~cm})$. b Example spectra. The black line represents averaged spectra, the red line is an overlay of the spectral fit, the bottom line is the residual. Cho choline, $\mathrm{Cr}$ creatine, Glx glutamate + glutamine, NAA N-acetyl-aspartate, ppm parts per million.

derived from in vitro and in vivo metabolite spectra was included in the model, as reported elsewhere ${ }^{40}$. Exclusion criteria were (1) line width of the magnitude signal during manual shimming $>20 \mathrm{~Hz}$ at FWHM, (2) failure of fitting algorithm, and (3) Cramer-Rao lower bounds (CRLB) > $20 \%$. Metabolites were quantified with respect to internal water (for comparison purposes also with respect to creatine). Structural scans were segmented into gray matter (GM), white matter (WM), and cerebrospinal fluid to calculate voxel tissue content, using in house Matlab codes.

\section{Statistical analyses}

SAS version 9.4 (Cary, NC) was used for statistical analyses. Mixed effect repeated measures linear models were used to investigate the effects of group and time on neurometabolites. Voxel GM fraction (GM\%/GM\% + WM $\%{ }^{41}$ ) was also included as a covariate in the model. Both a model assuming a common variance for $\mathrm{HC}$ and $\mathrm{SZ}$, as well as a model that allowed for separate variances for $\mathrm{HC}$ and SZ were considered. Akaike's information criterion was used to select the final model. A $p$ value of $<$ 0.05 was considered statistically significant.

In an exploratory fashion we also examined relationships between clinical characteristics (RBANS, BPRS, treatment response [defined as \% decrease in BPRS total scores after 16 weeks of treatment], log transformed duration of untreated psychosis) and metabolites using partial correlations with age and gray matter fraction as covariates.

\section{Results}

\section{Sample characteristics and demographics}

Of the 29 FEP enrolled in this study, five dropped before the baseline scans were completed (four could not tolerate the scanning environment, one withdrew consent prior to scanning), one was excluded from all analyses due to gross anatomical abnormalities observed at the baseline anatomical scan, five dropped prior to the week 6 scan (one subject became pregnant, one moved out of state, one was lost to follow-up, one withdrew consent, one developed a neurological condition), and one subject did not tolerate the scan at week 16 . In addition, MRS data were not obtained for three subjects at baseline, for three subjects at week 6 , and for one subject at week 16 (either because of poor shimming quality or subject request to terminate the scan before obtaining the MPFC MRS scan) in this multimodal neuroimaging study.

To obtain normative data, 22 matched HC were scanned at the same three time points as the FEP patients with an average inter-scan interval of $49.5+/-13.6$ days between the first and second scan (corresponding to baseline and week 6 scan in FEP), and $71.3+/-12.6$ days between the second and third scan (corresponding to week 6 and week 16 scan in FEP). One HC was lost to follow-up after the first scan, and one was withdrawn from the study because they did not tolerate the MRI scan. In addition, MRS data were not obtained for two subjects and a water unsuppressed scan was not obtained for one subject at baseline, for two subjects at week 6 , and for seven subjects at week 16 in this multimodal neuroimaging study.

In summary, quantifiable MRS data for the MPFC were available for $18 \mathrm{HC}$ and $20 \mathrm{FEP}$ at baseline, for $19 \mathrm{HC}$ and 15 FEP at week 6 , and for $14 \mathrm{HC}$ and 16 FEP at week 16 . For one FEP Cho and $\mathrm{Cr}$ values at week 6 were excluded because of our MRS data quality assessment criteria.

$\mathrm{HC}$ and FEP did not differ in gender, age, or parental socioeconomic status. As expected, RBANS scores were significantly lower in FEP compared with HC (Table 1). 
Table 1 Demographics and clinical measures ${ }^{a}$.

\begin{tabular}{|c|c|c|c|c|}
\hline & $\mathrm{HC}(n=21)$ & $\operatorname{FEP}(n=22)$ & $t / X^{2}$ & $p$ value \\
\hline Gender (\% male) & 62 & 64 & 0.01 & 0.91 \\
\hline Age & $22.95(5.01)$ & $22.73(5.52)$ & 0.14 & 0.89 \\
\hline Parental occupation ${ }^{b}$ & $3.95(3.15)$ & $4.95(5.00)$ & -0.78 & 0.44 \\
\hline $\begin{array}{l}\text { Smoking status (\% } \\
\text { smoker) }\end{array}$ & 0 & 32 & 7.98 & $<0.01$ \\
\hline \multicolumn{5}{|l|}{ Diagnosis } \\
\hline Schizophrenia & & 15 & & \\
\hline Schizoaffective disorder & & 5 & & \\
\hline Unspecified psychosis & & 2 & & \\
\hline $\begin{array}{l}\text { Duration of untreated } \\
\text { psychosis (mean; } \\
\text { median; [range] in weeks) }\end{array}$ & & $\begin{array}{l}124.55 ; 45 ; \\
{[1-626]}\end{array}$ & & \\
\hline $\begin{array}{l}\text { Risperidone dose week } 6 \\
\text { (mean; median; } \\
\text { [range] in } \mathrm{mg} \text { ) }\end{array}$ & & $3.61 ; 4 ;[1-8]$ & & \\
\hline $\begin{array}{l}\text { Risperidone dose at } \\
\text { week } 16 \text { (mean, SD; } \\
\text { median; [range] in mg) }\end{array}$ & & $4.65 ; 4 ;[0-8]$ & & \\
\hline \multicolumn{5}{|l|}{ BPRS $^{d}$} \\
\hline \multicolumn{5}{|l|}{ Baseline } \\
\hline Total & & $50.91(9.41)$ & & \\
\hline Positive & & $11.55(3.42)$ & & \\
\hline Negative & & $7.41(3.57)$ & & \\
\hline \multicolumn{5}{|l|}{ Week 6} \\
\hline Total & & $34.00(10.88)$ & & \\
\hline Positive & & $5.67(2.61)$ & & \\
\hline $\begin{array}{l}\text { Negative } \\
\text { Week } 16\end{array}$ & & $6.17(2.83)$ & & \\
\hline Total & & $34.44(11.44)$ & & \\
\hline Positive & & $5.44(2.9)$ & & \\
\hline Negative & & $6.94(2.82)$ & & \\
\hline \multicolumn{5}{|l|}{ RBANS $^{e}$} \\
\hline Total index & $91.14(13.32)$ & $70.42(14.61)$ & 4.69 & $<0.01$ \\
\hline Immediate memory & $100.67(15.57)$ & $78.95(16.7)$ & 4.26 & $<0.01$ \\
\hline Visuospatial & $85.62(13.67)$ & $74.58(15.21)$ & 2.42 & 0.02 \\
\hline Language & $94.81(17.06)$ & 81.79 (12.49) & 2.73 & $<0.01$ \\
\hline Attention span & $94.62(19.05)$ & $75.16(18.97)$ & 3.23 & $<0.01$ \\
\hline Delayed memory & $91.81(8.37)$ & $71.53(20.14)$ & 4.23 & $<0.01$ \\
\hline
\end{tabular}

FEP first-episode psychosis, $H C$ healthy control.

${ }^{a}$ Mean (SD) unless indicated otherwise.

${ }^{\mathrm{b}}$ Ranks determined from Diagnostic Interview for Genetic Studies (1-18 scale); higher rank (lower numerical value) corresponds to higher socioeconomic status.

'Data on duration of untreated psychosis were not available for two patients. ${ }^{\mathrm{d} B r i e f}$ Psychiatric Rating Scale (1-7 scale); positive (conceptual disorganization, hallucinatory behavior, and unusual thought content); negative (emotional withdrawal, motor retardation, and blunted affect).

${ }^{d}$ Repeatable Battery for the Assessment of Neuropsychological Status, data not available for three FEP.

\section{MRS measurements}

Of the models for mixed effects repeated measures considered, the model assuming a common variance for $\mathrm{HC}$ and FEP had the best fit. After controlling for time and GM fraction, no significant differences in metabolites between groups were found, no group by time interaction was present ( $p>0.2$ for all; Table 2, Fig. 2). We also found no differences between metabolites when using creatine instead of internal water as a reference (Table 3).

We found no correlations between neurometabolite levels and BPRS scores, RBANS scores, or duration of untreated psychosis.

\section{Discussion}

Here we conducted a longitudinal MRS study prospectively examining the effects of antipsychotic treatment on glutamatergic measures in the MPFC in a group of medication-naive FEP patients. We did not find any baseline metabolite alterations in patients compared with HC. Interestingly, metabolite levels were not affected by medications both after 6 and 16 weeks of treatment with risperidone.

We found no increase in Glx in medication-naive FEP, which stands in contrast with prior findings by Kegeles et al. who showed elevated GABA and Glx in the MPFC in unmedicated schizophrenia patients ${ }^{22}$, and Chen et al. who reported elevated glutamate and reduced glutamine in the ventromedial prefrontal cortex in FEP who had been off antipsychotic medications for at least 2 weeks ${ }^{23}$. Examining glutamatergic measures in the MPFC across illness stages, Natsubori and colleagues reported no alterations in Glx in the MPFC in subjects at ultrahigh risk for schizophrenia or FEP patients with no more than 16 weeks of cumulative antipsychotic medication exposure, but did report a decrease in Glx in chronic schizophrenia patients ${ }^{42}$. In contrast, Wang and colleagues did not find alterations in Glx in ultrahigh risk subjects, but reported reduced MPFC Glx in a small group of medication-naive $\mathrm{FEP}^{43}$. It stands to reason that illness stage could, at least in part, account for discrepancies in findings. Furthermore, age effects and effects of prior medication exposure in studies before undergoing a medication washout may contribute to discrepancies in findings, where patients in early illness stages may be more prone to glutamatergic elevations than those in the chronic stages ${ }^{44}$.

In a similarly designed study, de la Fuente-Sandoval and colleagues enrolled 28 antipsychotic medication-naive FEP patients and assessed MPFC Glx before and after 4 weeks of treatment with risperidone ${ }^{36}$. In contrast to us, they report that elevated Glx was seen in patients at baseline, but no longer was present after treatment. Interestingly, the duration of untreated psychosis in this group was 52 weeks on average (only two subjects had experienced psychotic symptoms for more than 2 years), while in our sample it was $\sim 2.4$ years on average. Furthermore, the mean daily dose of risperidone in this sample was about $25 \%$ lower than in our sample, suggesting that the patients in our sample had an overall higher psychosis burden. It is possible that these important clinical differences could have contributed to 
Table 2 Neurometabolites, spectral quality, and tissue fraction.

\begin{tabular}{|c|c|c|c|c|}
\hline & $\mathrm{HC}$ & FEP & $\mathrm{F} / \mathrm{t}$ & $p$ \\
\hline \multicolumn{5}{|l|}{ Neurometabolites $^{\mathrm{a}}$} \\
\hline \multicolumn{5}{|l|}{ Baseline } \\
\hline NAA & $0.38(0.05)$ & $0.37(0.06)$ & 0.29 & 0.77 \\
\hline $\mathrm{Glx}$ & $0.22(0.05)$ & $0.22(0.06)$ & 0.08 & 0.94 \\
\hline Cho & $0.16(0.03)$ & $0.17(0.04)$ & -0.1 & 0.92 \\
\hline $\mathrm{Cr}$ & $0.28(0.06)$ & $0.28(0.08)$ & -0.25 & 0.80 \\
\hline \multicolumn{5}{|l|}{ Week 6} \\
\hline NAA & $0.39(0.04)$ & $0.37(0.03)$ & 1.05 & 0.30 \\
\hline Glx & $0.23(0.05)$ & $0.22(0.03)$ & 0.66 & 0.52 \\
\hline Cho & $0.16(0.02)$ & $0.17(0.03)$ & -0.94 & 0.35 \\
\hline $\mathrm{Cr}$ & $0.28(0.06)$ & $0.29(0.04)$ & -0.43 & 0.67 \\
\hline \multicolumn{5}{|l|}{ Week 16} \\
\hline NAA & $0.39(0.04)$ & $0.39(0.04)$ & -0.18 & 0.86 \\
\hline Glx & $0.22(0.07)$ & $0.23(0.03)$ & -0.53 & 0.60 \\
\hline Cho & $0.17(0.03)$ & $0.18(0.02)$ & -1.19 & 0.24 \\
\hline $\mathrm{Cr}$ & $0.29(0.05)$ & $0.30(0.06)$ & -0.23 & 0.82 \\
\hline \multicolumn{5}{|c|}{ Spectral quality indices } \\
\hline \multicolumn{5}{|l|}{ Baseline } \\
\hline NAA CRLB & $1.50(0.20)$ & $1.70(0.44)$ & -1.88 & 0.07 \\
\hline Glx CRLB & $9.16(1.70)$ & $10.44(3.07)$ & -1.61 & 0.12 \\
\hline Cho CRLB & $2.77(0.45)$ & $3.24(1.41)$ & -1.40 & 0.18 \\
\hline $\mathrm{Cr}$ CRLB & $1.77(0.28)$ & $2.01(0.71)$ & -1.31 & 0.20 \\
\hline FWHM & $6.46(2.16)$ & $6.86(1.59)$ & -0.66 & 0.52 \\
\hline SNR & $15.18(2.31)$ & $13.72(3.73)$ & -1.46 & 0.15 \\
\hline \multicolumn{5}{|l|}{ Week 6} \\
\hline NAA CRLB & $1.54(0.30)$ & $1.55(0.22)$ & -0.21 & 0.84 \\
\hline Glx CRLB & $10.16(3.47)$ & $9.02(1.86)$ & 1.21 & 0.24 \\
\hline Cho CRLB & $2.87(0.55)$ & $2.68(0.34)$ & 1.12 & 0.27 \\
\hline Cr CRLB & $1.84(0.40)$ & $1.88(0.63)$ & -0.27 & 0.79 \\
\hline FWHM & $7.60(1.96)$ & $6.49(1.00)$ & 2.15 & 0.04 \\
\hline SNR & $16.09(2.35)$ & $14.37(1.42)$ & 2.67 & 0.01 \\
\hline \multicolumn{5}{|l|}{ Week 16} \\
\hline NAA CRLB & $1.54(0.23)$ & $1.56(0.27)$ & -0.27 & 0.79 \\
\hline Glx CRLB & $10.90(3.56)$ & $9.81(2.40)$ & 1.00 & 0.33 \\
\hline Cho CRLB & $2.83(0.30)$ & $2.95(1.25)$ & -0.34 & 0.73 \\
\hline Cr CRLB & $1.79(0.24)$ & $2.04(1.46)$ & -0.64 & 0.53 \\
\hline FWHM & 7.99 (2.55) & $7.50(1.63)$ & 0.63 & 0.53 \\
\hline SNR & $16.09(3.17)$ & $15.39(2.01)$ & 0.74 & 0.47 \\
\hline \multicolumn{5}{|l|}{ Voxel tissue fraction } \\
\hline \multicolumn{5}{|l|}{ Baseline } \\
\hline Gray matter (\%) & 74.35 (5.59) & 75.88 (3.88) & -1.01 & 0.32 \\
\hline White matter (\%) & $11.08(7.71)$ & $9.74(3.49)$ & 0.71 & 0.48 \\
\hline CSF $(\%)$ & $14.78(3.17)$ & $13.85(2.90)$ & 0.97 & 0.34 \\
\hline \multicolumn{5}{|l|}{ Week 6} \\
\hline Gray matter (\%) & $76.25(2.81)$ & $75.45(2.84)$ & 0.84 & 0.41 \\
\hline White matter (\%) & $9.07(2.57)$ & $9.16(2.36)$ & -0.11 & 0.91 \\
\hline CSF $(\%)$ & $14.82(2.72)$ & $15.45(3.07)$ & -0.64 & 0.53 \\
\hline \multicolumn{5}{|l|}{ Week 16} \\
\hline Gray matter (\%) & $75.30(2.95)$ & $76.18(4.05)$ & -0.69 & 0.49 \\
\hline White matter (\%) & $10.58(3.24)$ & $9.07(2.38)$ & 1.52 & 0.14 \\
\hline CSF (\%) & $14.00(2.15)$ & $14.21(3.59)$ & -0.23 & 0.82 \\
\hline
\end{tabular}

HC healthy control, FEP first-episode psychosis patient, NAA N-acetyl-aspartate, Glx glutamate + glutamine, Cho choline, $C r$ creatine, CRLB Cramer-Rao lower bounds, FWHM full width half maximum, SNR signal-to-noise ratio, CSF cerebrospinal fluid.

anstitutional units. discrepancies in findings. Future studies investigating the impact of duration of untreated psychosis on glutamatergic indices will be informative in this regard.

Another consideration are differences of acquisition techniques across various studies. While we used PRESS, Kegeles and colleagues used MEGA-PRESS to quantify Glx. A technical report comparing these acquisition sequences recently reported that spectral values for Glx acquired with a $\mathrm{TE}=80 \mathrm{~ms}$ PRESS sequence correspond to the off-resonance but not the difference spectra acquired with a MEGA-PRESS sequence in healthy volunteers and medicated FEP subjects $^{45}$. However, as the authors underscore, it remains an open question whether glutamatergic values from PRESS spectra are more or less valid than glutamatergic values from MEGA-PRESS difference spectra. A number of studies relied on shorter TE and spectral fitting to derive the levels of glutamatergic compounds that may explain some of the discrepancies. Last, glutamatergic alterations may vary depending on the region of interest studied, with most studies targeting the frontal regions, specifically the MPFC reporting elevations in glutamatergic compounds, while many other studies targeting more dorsal brain regions, e.g., the dorsal anterior cingulate cortex, found no elevations ${ }^{46}$.

Contrary to our hypothesis, we observed no decrease in Glx after 6 or 16 weeks of treatment with risperidone. The lack of changes in metabolite levels stands in contrast with a literature review suggesting that antipsychotic medications may affect glutamate levels, with an estimated $6.5 \%$ reduction in Glx across brain regions ${ }^{27}$. However, several studies report no antipsychotic induced changes $^{31}$, or regionally selective reductions ${ }^{29}$ in glutamatergic measurements. It is possible that variable antipsychotic medications have diverse effects on the glutamate system. Investigating differential drug effects on brain metabolite levels in an animal model, McLoughlin and colleagues report antipsychotic induced reductions of glutamate in the frontal cortex only with clozapine and olanzapine, but not risperidone, haloperidol, or aripiprazole ${ }^{47}$. This is further supported by experiments with the NMDA receptor blocker ketamine, a popular pharmacological model of psychosis ${ }^{11,12,48-53}$, showing that pretreatment with high dose olanzapine, but not risperidone attenuates ketamine induced alterations in brain metabolism ${ }^{54}$.

\section{Strengths and limitations}

Our findings have to be interpreted in context of a number of strengths and limitations. We enrolled exclusively medication-naive FEP and a group of $\mathrm{HC}$ matched on key demographic characteristics in this prospective longitudinal trial. Even though smoking rates differed between groups, metabolites did not. To minimize 


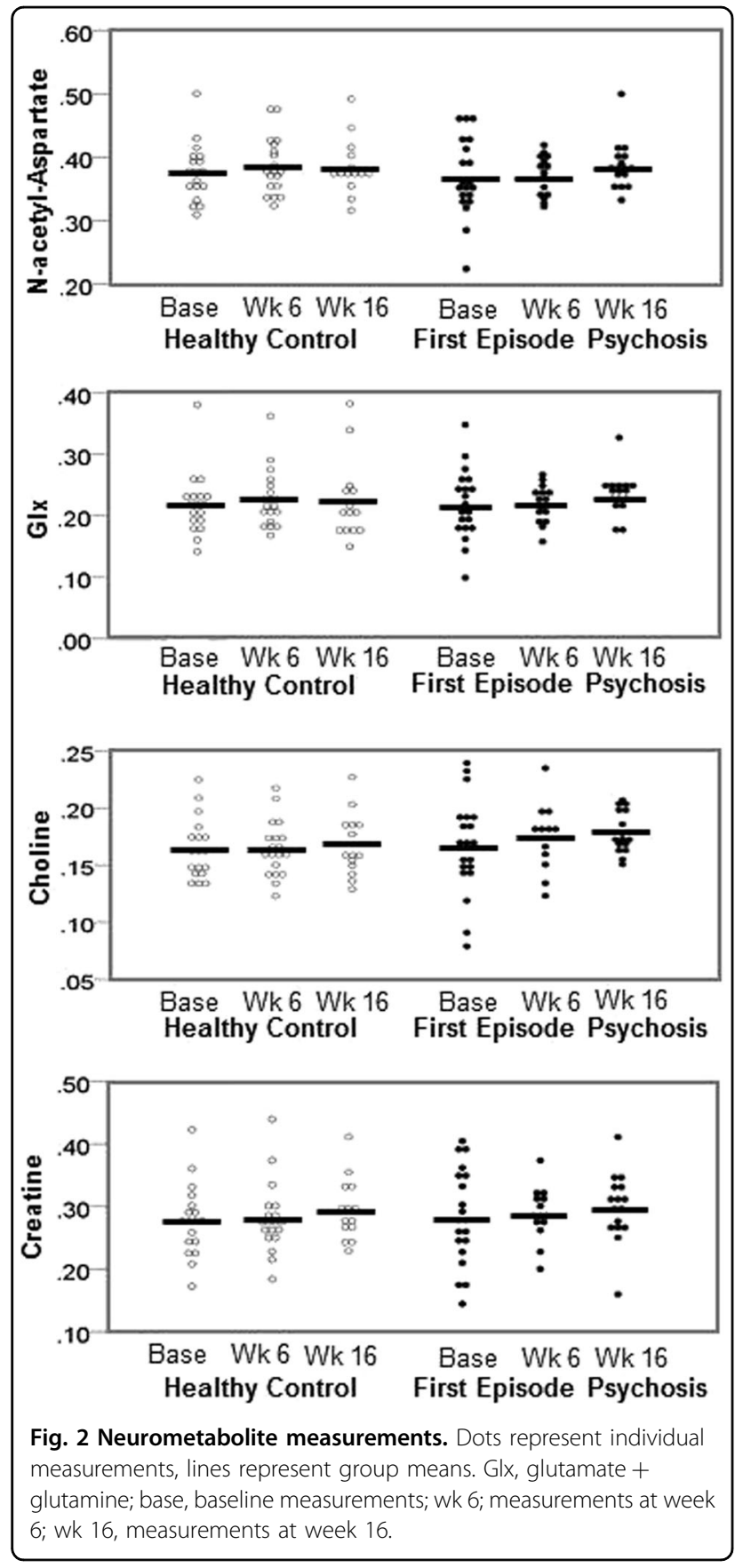

variance in the data, we used a single antipsychotic medication. We assessed adherence using pill counts and self-report, but future studies would benefit from monitoring risperidone blood levels to confirm compliance. Because it is ethically not permissible to withhold known effective medications from patients, we did not include a placebo group. On a technical note, we used a relatively long TE of $80 \mathrm{~ms}$ for MRS in an effort to optimize the glutamate signal $^{39}$ and minimize macromolecule contribution, but this imparts appreciable J-modulation and
Table 3 Neurometabolite concentrations, with creatine as reference.

\begin{tabular}{lllll}
\hline & HC & FEP & $\boldsymbol{t}$ & $\boldsymbol{p}$ \\
\hline Neurometabolites $^{\mathrm{a}}$ & & & & \\
Baseline & & & & \\
NAA/Cr & $1.55(0.28)$ & $1.48(0.25)$ & 0.72 & 0.48 \\
$\mathrm{Glx} / \mathrm{Cr}$ & $0.84(0.16)$ & $0.81(0.11)$ & 0.70 & 0.49 \\
Cho/Cr & $0.73(0.17)$ & $0.74(0.10)$ & -0.14 & 0.89 \\
Week 6 & & & & \\
NAA/Cr & $1.57(0.22)$ & $1.49(0.26)$ & 0.87 & 0.39 \\
Glx/Cr & $0.87(0.10)$ & $0.83(0.13)$ & 0.96 & 0.35 \\
Cho/Cr & $0.72(0.15)$ & $0.73(0.09)$ & -0.04 & 0.97 \\
Week 16 & & & & \\
NAA/Cr & $1.48(0.11)$ & $1.41(0.13)$ & 1.48 & 0.15 \\
Glx/Cr & $0.80(0.10)$ & $0.81(0.09)$ & -0.30 & 0.77 \\
Cho/Cr & $0.70(0.08)$ & $0.71(0.08)$ & -0.43 & 0.67 \\
\hline
\end{tabular}

HC healthy control, FEP first-episode psychosis patient, NAA N-acetyl-aspartate, Glx glutamate + glutamine, Cho choline, $\mathrm{Cr}$ creatine.

T2 relaxation effects on the spectrum, and the water signal is highly $\mathrm{T} 2$ weighted and very sensitive to cerebrospinal fluid contamination, as well as partial volume effects. Last, it is possible that we did not have sufficient power to detect group differences in Glx or changes in Glx with antipsychotic treatment, as prior studies have suggested that at least 24 subjects in each group may be necessary to detect a reduction in Glx with antipsychotic treatment with $80 \%$ power $^{27}$. The limited sample size reflects the difficulty in recruiting this patient population. Because of this, we were unable to dichotomize patients into good and poor treatment responders to determine if reductions in Glx are more likely in those with symptomatic improvement as recently reported by Merritt and colleagues $^{55}$.

\section{Conclusions}

The underlying mechanisms and biological correlates of short and longer treatment trails remain largely unknown, and it is possible that longer treatment durations may be needed to detect treatment-related changes in neurometabolites. In this prospective MRS study, we examined Glx before treatment was started, after 6 and again after 16 weeks of treatment, but failed to detect changes in metabolite levels.

In summary, our study adds to the efforts in better understanding glutamatergic neurometabolism in schizophrenia, especially as it relates to antipsychotic exposure. It will be critically important to systematically investigate glutamatergic measurements across various 
areas of the brain, different illness stages, and different medication statuses to improve our mechanistic understanding and facilitate biomarker development.

\section{Acknowledgements}

We would like to thank the UAB neuroimaging community for their support, especially their flexibility in making same day scanning for this protocol possible. This work was supported by the National Institute of Mental Health (R01MH081014 and R01MH102951, A.C.L.; K23MH106683, N.V.K.). A.C.L. has received an investigator initiated grant from Janssen Pharmaceuticals.

\section{Author details}

'Department of Psychiatry and Behavioral Neurobiology, University of Alabama at Birmingham, Birmingham, AL, USA. ${ }^{2}$ Department of Neurology, University of Alabama at Birmingham, Birmingham, AL, USA. ${ }^{3}$ Department of Neurology, Birmingham VA Medical Center, Birmingham, AL, USA. ${ }^{4}$ MRI Research Center, Department of Electrical and Computer Engineering, Auburn University, Auburn, AL, USA

\section{Conflict of interest}

The authors declare that they have no conflict of interest.

\section{Publisher's note}

Springer Nature remains neutral with regard to jurisdictional claims in published maps and institutional affiliations.

Received: 22 March 2019 Revised: 25 November 2019 Accepted: 8 December 2019

Published online: 21 January 2020

\section{References}

1. Kreyenbuhl, J., Buchanan, R. W., Dickerson, F. B. \& Dixon, L. B. Schizophrenia Patient Outcomes Research T. The Schizophrenia Patient Outcomes Research Team (PORT): updated treatment recommendations 2009. Schizophr. Bull. 36, 94-103 (2010).

2. Lehman, A. F. et al. Practice guideline for the treatment of patients with schizophrenia, second edition. Am. J. Psychiatry 161(2 Suppl), 1-56 (2004).

3. Remington, G. et al. Guidelines for the pharmacotherapy of schizophrenia in adults. Can. J. Psychiatry 62, 604-616 (2017).

4. Barnes, T. R. Schizophrenia Consensus Group of British Association for P. Evidence-based guidelines for the pharmacological treatment of schizophrenia: recommendations from the British Association for Psychopharmacology. J. Psychopharmacol. 25, 567-620 (2011).

5. Kane, J. M., Leucht, S., Carpenter, D. \& Docherty, J. P. Expert Consensus Panel for Optimizing Pharmacologic Treatment of Psychotic D. The expert consensus guideline series. Optimizing pharmacologic treatment of psychotic disorders. Introduction: methods, commentary, and summary. J. Clin. Psychiatry 64(Suppl 12), 5-19 (2003).

6. Agid, O., Kapur, S., Arenovich, T. \& Zipursky, R. B. Delayed-onset hypothesis of antipsychotic action: a hypothesis tested and rejected. Arch. Gen. Psychiatry 60 , 1228-1235 (2003)

7. Leucht, S., Busch, R., Hamann, J., Kissling, W. \& Kane, J. M. Early-onset hypothesis of antipsychotic drug action: a hypothesis tested, confirmed and extended. Biol. Psychiatry 57, 1543-1549 (2005).

8. Gallego, J. A. et al. Time to treatment response in first-episode schizophrenia: should acute treatment trials last several months? J. Clin. Psychiatry 72, 1691-1696 (2011).

9. Kraguljac, N. V. et al. Neurometabolites in schizophrenia and bipolar disorder -a systematic review and meta-analysis. Psychiatry Res. 203, 111-125 (2012).

10. Olney, J. W. \& Farber, N. B. Glutamate receptor dysfunction and schizophrenia. Arch. Gen. Psychiatry 52, 998-1007 (1995).

11. Lahti, A. C., Weiler, M. A., Tamara Michaelidis, B. A., Parwani, A. \& Tamminga, C. A. Effects of ketamine in normal and schizophrenic volunteers. Neuropsychopharmacology 25, 455-467 (2001).

12. Kraguljac, N. V. et al. Ketamine modulates hippocampal neurochemistry and functional connectivity: a combined magnetic resonance spectroscopy and resting-state fMRI study in healthy volunteers. Mol. Psychiatry 22, 562-569 (2017).

13. Moghaddam, B. \& Javitt, D. From revolution to evolution: the glutamate hypothesis of schizophrenia and its implication for treatment. Neuropsychopharmacology 37, 4-15 (2012).

14. Javitt, D. C. Twenty-five years of glutamate in schizophrenia: are we there yet? Schizophr. Bull. 38, 911-913 (2012).

15. Lodge, D. J. \& Grace, A. A. Hippocampal dysregulation of dopamine system function and the pathophysiology of schizophrenia. Trends Pharm. Sci. 32, 507-513 (2011).

16. Floresco, S. B., Todd, C. L. \& Grace, A. A. Glutamatergic afferents from the hippocampus to the nucleus accumbens regulate activity of ventral tegmental area dopamine neurons. J. Neurosci. 21, 4915-4922 (2001).

17. Grace, A. A. Dysregulation of the dopamine system in the pathophysiology of schizophrenia and depression. Nat. Rev. Neurosci. 17, 524-532 (2016).

18. Kraguljac, N. V., Reid, M. A., White, D. M., den Hollander, J. \& Lahti, A. C. Regional decoupling of $\mathrm{N}$-acetyl-aspartate and glutamate in schizophrenia. Neuropsychopharmacology 37, 2635-2642 (2012).

19. Sivaraman, S. et al. Neurometabolic abnormalities in the associative striatum in antipsychotic-naive first episode psychosis patients. Psychiatry Res. Neuroimaging. 281, 101-106 (2018).

20. Coughlin, J. M. et al. Decoupling of N-acetyl-aspartate and glutamate within the dorsolateral prefrontal cortex in schizophrenia. Curr. Mol. Med. 15, 176-183 (2015).

21. de la Fuente-Sandoval, C. et al. Higher levels of glutamate in the associative-striatum of subjects with prodromal symptoms of schizophrenia and patients with first-episode psychosis. Neuropsychopharmacology 36, 1781-1791 (2011).

22. Kegeles, L. S. et al. Elevated prefrontal cortex gamma-aminobutyric acid and glutamate-glutamine levels in schizophrenia measured in vivo with proton magnetic resonance spectroscopy. Arch. Gen. Psychiatry 69, 449-459 (2012).

23. Chen, T. et al. Abnormal concentration of GABA and glutamate in the prefrontal cortex in schizophrenia-an in vivo 1H-MRS Study. Shanghai Arch. Psychiatry 29, 277-286 (2017).

24. Hutcheson, N. L. et al. Multimodal analysis of the hippocampus in schizophrenia using proton magnetic resonance spectroscopy and functional magnetic resonance imaging. Schizophr. Res. 140, 136-142 (2012).

25. de la Fuente-Sandoval, $C$. et al. Glutamate levels in the associative striatum before and after 4 weeks of antipsychotic treatment in first-episode psychosis: a longitudinal proton magnetic resonance spectroscopy study. JAMA PSychiatry 70, 1057-1066 (2013).

26. Egerton, A. et al. Response to initial antipsychotic treatment in first episode psychosis is related to anterior cingulate glutamate levels: a multicentre $(1) \mathrm{H}-$ MRS study (OPTiMiSE). Mol. Psychiatry. 23, 2145-2155 (2018).

27. Egerton, A. et al. Effects of antipsychotic administration on brain glutamate in schizophrenia: a systematic review of longitudinal (1)H-MRS studies. Front Psychiatry 8, 66 (2017).

28. Goto, N. et al. Six-month treatment with atypical antipsychotic drugs decreased frontal-lobe levels of glutamate plus glutamine in early-stage firstepisode schizophrenia. Neuropsychiatr. Dis. Treat. 8, 119-122 (2012).

29. Szulc, A. et al. Proton magnetic resonance spectroscopy study of brain metabolite changes after antipsychotic treatment. Pharmacopsychiatry $\mathbf{4 4}$ 148-157 (2011).

30. Aoyama, N. et al. Grey matter and social functioning correlates of glutamatergic metabolite loss in schizophrenia. Br. J. Psychiatry 198, 448-456 (2011).

31. Kraguljac, N. V. et al. A longitudinal magnetic resonance spectroscopy study investigating effects of risperidone in the anterior cingulate cortex and hippocampus in schizophrenia. Schizophr. Res. 210, 239-244 (2019).

32. de la Fuente-Sandoval, C. et al. Striatal glutamate and the conversion to psychosis: a prospective 1H-MRS imaging study. Int J. Neuropsychopharmacol. 16, 471-475 (2013).

33. Smesny, S. et al. Glutamatergic dysfunction linked to energy and membrane lipid metabolism in frontal and anterior cingulate cortices of never treated first-episode schizophrenia patients. Schizophr. Res. 168, 322-329 (2015).

34. Theberge, J. et al. Glutamate and glutamine measured with $4.0 \mathrm{~T}$ proton MRS in never-treated patients with schizophrenia and healthy volunteers. Am. J. Psychiatry 159, 1944-1946 (2002).

35. Bartha, R. et al. Measurement of glutamate and glutamine in the medial prefrontal cortex of never-treated schizophrenic patients and healthy controls by proton magnetic resonance spectroscopy. Arch. Gen. Psychiatry $\mathbf{5 4}$ 959-965 (1997). 
36. de la Fuente-Sandoval, C. et al. Prefrontal and striatal gamma-aminobutyric acid levels and the effect of antipsychotic treatment in first-episode psychosis patients. Biol. Psychiatry 83, 475-483 (2018).

37. Nurnberger, J. I. Jr et al. Diagnostic interview for genetic studies. Rationale, unique features, and training. NIMH Genetics Initiative. Arch. Gen. Psychiatry 51, 849-859 (1994). discussion 63-64.

38. Woerner, M. G., Mannuzza, S. \& Kane, J. M. Anchoring the BPRS: an aid to improved reliability. Psychopharmacol. Bull. 24, 112-117 (1988).

39. Schubert, F., Gallinat, J., Seifert, F. \& Rinneberg, H. Glutamate concentrations in human brain using single voxel proton magnetic resonance spectroscopy at 3 Tesla. Neuroimage 21, 1762-1771 (2004).

40. Reid, M. A. et al. Assessments of function and biochemistry of the anterior cingulate cortex in schizophrenia. Biol. Psychiatry 68, 625-633 (2010).

41. Caprihan, A. et al. The paradoxical relationship between white matter, psychopathology and cognition in schizophrenia: a diffusion tensor and proton spectroscopic imaging study. Neuropsychopharmacology 40, 2248-2257 (2015).

42. Natsubori, T. et al. Reduced frontal glutamate + glutamine and $\mathrm{N}$ acetylaspartate levels in patients with chronic schizophrenia but not in those at clinical high risk for psychosis or with first-episode schizophrenia. Schizophr. Bull. 40, 1128-1139 (2014).

43. Wang, J. et al. Reduced gamma-aminobutyric acid and glutamate+glutamine levels in drug-naive patients with first-episode schizophrenia but not in those at ultrahigh risk. Neural Plast. 2016, 3915703 (2016).

44. Iwata, Y. et al. Glutamatergic neurometabolite levels in patients with ultra-treatment-resistant schizophrenia: a cross-sectional $3 T$ proton magnetic resonance spectroscopy study. Biol. Psychiatry 85, 596-605 (2019).

45. Maddock, R. J., Caton, M. D. \& Ragland, J. D. Estimating glutamate and Glx from GABA-optimized MEGA-PRESS: off-resonance but not difference spectra values correspond to PRESS values. Psychiatry Res. Neuroimaging. 279, 22-30 (2018)

46. Iwata, Y. et al. Neurometabolite levels in antipsychotic-naive/free patients with schizophrenia: A systematic review and meta-analysis of
(1)H-MRS studies. Prog. Neuropsychopharmacol. Biol. Psychiatry 86 340-352 (2018).

47. McLoughlin, G. A. et al. Analyzing the effects of psychotropic drugs on metabolite profiles in rat brain using 1H NMR spectroscopy. J. Proteome Res. 8 , 1943-1952 (2009).

48. Lahti, A. C., Holcomb, H. H., Medoff, D. R. \& Tamminga, C. A. Ketamine activates psychosis and alters limbic blood flow in schizophrenia. Neuroreport 6, 869-872 (1995).

49. Lahti, A. C., Koffel, B., LaPorte, D. \& Tamminga, C. A. Subanesthetic doses of ketamine stimulate psychosis in schizophrenia. Neuropsychopharmacology 13 9-19 (1995).

50. Kraguljac, N. V. et al. Mnemonic discrimination deficits in first-episode psychosis and a ketamine model suggests dentate gyrus pathology linked to $\mathrm{N}$ methyl-D-aspartate receptor hypofunction. Biol. Psychiatry Cogn. Neurosci. Neuroimaging. 3, 231-238 (2018).

51. Rowland, L. M. et al. Effects of ketamine on anterior cingulate glutamate metabolism in healthy humans: a 4-T proton MRS study. Am. J. Psychiatry 162, 394-396 (2005)

52. Stone, J. M. et al. Ketamine effects on brain GABA and glutamate levels with $1 \mathrm{H}-\mathrm{MRS}$ : relationship to ketamine-induced psychopathology. Mol. Psychiatry 17, 664-665 (2012).

53. Holcomb, H. H., Lahti, A. C., Medoff, D. R., Weiler, M. \& Tamminga, C. A Sequential regional cerebral blood flow brain scans using PET with $\mathrm{H} 2(15) \mathrm{O}$ demonstrate ketamine actions in CNS dynamically. Neuropsychopharmacology 25, 165-172 (2001).

54. Duncan, G. E., Miyamoto, S., Leipzig, J. N. \& Lieberman, J. A. Comparison of the effects of clozapine, risperidone, and olanzapine on ketamine-induced alterations in regional brain metabolism. J. Pharm. Exp. Ther. 293, 8-14 (2000).

55. Merritt, K. et al. Remission from antipsychotic treatment in first episode psychosis related to longitudinal changes in brain glutamate. NPJ Schizophr. 5, 12 (2019). 